\title{
Violencia, crisis del capitalismo global y juventud
}

\author{
/ Niolence crisis of global \\ capitalism and youth
}

\section{//Violência, crise do capitalismo global e juventude}

\begin{tabular}{l|l} 
Reflexiones & $\begin{array}{l}\text { Revista Colombiana } \\
\text { de Educación, N. 62. } \\
\text { Primer semestre de 2012, } \\
\text { Bogotá, Colombia. }\end{array}$
\end{tabular}
Bárbara Yadira García Sánchez * Javier Guerrero Barón **

\section{Resumen}

\begin{abstract}
Doctora en Ciencias de la Educación de la Red de Universidades del Doctorado en Ciencias de la Educación, magister en Sociología de la Cultura de la Universidad Nacional de Colombia, especialista en Gobierno Escolar de la Universidad El Bosque, socióloga de la Universidad Cooperativa de Colombia y licenciada en Administración Educativa de la Universidad de La Sabana. Docente-investigadora del Doctorado Interinstitucional en Educación, Universidad Distrital Francisco José de Caldas, vinculada al grupo de investigación Formación de Educadores. Correo electrónico: barbaragarciasanchez@yahoo.com

Doctor y magister en Historia y sociólogo de la Universidad Nacional de Colombia. Director del Doctorado en Historia de la Universidad Pedagógica y Tecnológica de Colombia-UPTC, vinculado al grupo de investigación Conflictos Sociales Siglo XX. Correo electrónico: javier. guerrero@uptc.edu.co
\end{abstract}

El presente artículo desarrolla algunas reflexiones que sirvieron de marco conceptual para la comprensión del contexto social en el que se articulan las relaciones de violencia en el mundo contemporáneo, las cuales fueron objeto de investigación en el proyecto Violencia escolar en Bogotá ${ }^{1}$, realizado con metodología Investigación-Acción-Participativa-IAP, aplicando el modelo "Núcleos de Educación Social $^{2}$ ", estudiando las estructuras sociales que generan violencias.

1 Proyecto de investigación: "Violencia escolar en Bogotá: Una mirada desde las familias, los maestros y los jóvenes. Aplicación de un modelo cualitativo en familia, escuela y barrio. Universidad Distrital, Universidad Pedagógica y Tecnológica de Colombia y Colciencias, convenio 382 de 2008".

2 Los Núcleos de Educación Social, NES, son pequeños grupos que se conforman en las comunidades escolares, barriales o familiares, con la participación de padres, madres, maestros, niños, niñas, adolescentes, agentes comunitarios o líderes barriales, entre otros, encaminados a adelantar procesos de investigación cualitativa con métodos participativos, desarrollando un proceso por etapas de investigación y escenarios de intervención con el propósito de conocer, observar, analizar, comprender, interpretar, prevenir y transformar problemáticas sociales que se presentan en las familias, las instituciones educativas o los barrios. La dinámica investigativa, preventiva, participativa, se inicia previa concertación con los participantes y con el acompañamiento de un docente, orientador o investigador durante el proceso.

\section{Palabras Clave}

Violencia, crisis social, capitalismo global, juventud. 


\section{Abstract}

This paper develops some reflections that aim to comprehend the social context where contemporary relationships of violence take place. This kind of relationships was the object of a research on scholar violence in Bogota (Colombia) ${ }^{3}$. The research used Participatory Action Research methodologies, and applied the "Nucleus of Social Education" Model to examine the social structures that generate violences (in plural) in five official educational institutions.

\section{Keywords}

Violence, social crisis, global capitalism, young

\section{Resumo}

O presente artigo desenvolve algumas reflexões que serviram de marco conceitual para a compreensão do contexto social no qual se articulam as relações de violência no mundo contemporâneo, as quais foram objeto de investigação no projeto "Violencia escolar em Bogotá", realizado com a metodologia Investigação-Ação-Participativa-IAP, aplicando-se o modelo "Núcleos de Educação Social", e estudando-se as estruturas sociais que geram violências.

\section{Palavras chave}

Violência, crise social, capitalismo global, juventude. 


\section{Violencia, crisis del capitalismo global y juventud}

\section{Introducción}

Este artículo parte de la reflexión sobre el capitalismo global o capitalismo posindustrial y su relación con las lógicas de violencia, exclusión y marginalidad que impacta fundamentalmente a la población, en especial a la infantil y juvenil, y asume al capitalismo global como el contexto general en el que se desatan las relaciones de violencia. La idea es examinar cómo el cambio súbito en un corto lapso ha impactado las relaciones interpersonales y la vida comunitaria, familiar y barrial de los sectores más pobres de la población.

El capitalismo global transita en oleadas por el mundo en tiempo real desde finales del siglo $\mathrm{XX}$, caminando de patria en patria en 24 horas, gracias a las redes telemáticas, arruinando el sector real de la economía a favor de los consorcios financieros totalmente dominados por los intereses transnacionales. Este fenómeno siempre estuvo presente en el siglo XX pero emergió con gran fuerza a finales de los años 80 y se catapultó en los 90 con la caída del muro y la implosión de la Unión Soviética, cuando los países Ilamados socialistas, incluida China, ingresaron a economías de mercado abierto y los países occidentales cayeron en una euforia desreguladora que entregó inconmensurables ventajas sobre los demás sectores de la economía a las corporaciones financieras, atacando directamente al mundo del trabajo, especialmente la estabilidad de todos los niveles de trabajadores, mediante mecanismos de flexibilidad laboral.

Hay un factor definitivo: en 1996 se pusieron a disposición del público y del comercio las redes de Internet, siendo apropiadas de manera inmediata por los establecimientos financieros, lo cual aceleró el proceso de internacionalización del capital. Estudios recientes reconocen en este hecho un componente fundamental para la explicación de este fenómeno, que luego se complementaría con la expansión de las redes sociales informáticas, causantes de transformaciones nunca vistas en la velocidad de difusión de los fenómenos económicos y sociales ${ }^{4}$.

En esta reflexión partimos de un hecho constatable: en tres décadas esta oleada destruyó lo que Bourdieu denominó "la civilización industrial", con cuyos mecanismos de redistribución social regulados por los Estados se había construido en cerca de tres siglos un correspondiente "Estado de Bienestar" que

4 Abundantes publicaciones y debates se producen en el mundo académico al respecto. El primer estudio empírico de consistencia que llamó la atención sobre el problema es la obra emblemática de Castells. Es importante recalcar que el primer tomo fue escrito en 1997 y la obra publicada en 1998, casi simultáneamente con los primeros impactos de "la sociedad red". Castells, M. (2006). La era de la información: Economía, sociedad y cultura. Vol. 3. México: Siglo XXI Editores. 
amortiguaba los efectos de la pobreza y el desarrollo desigual en esos países. Aunque el desarrollo de estas políticas fue casi incipiente en América Latina, algunos sectores tenían garantías de vida digna, y así grandes mayorías marginales debieran subsistir precariamente, había una mínima corresponsabilidad del capital y algunas garantías estatales para exigirla, y lo que hoy se denomina responsabilidad social de la empresa consistía en generar empleo y cumplir leyes y pactos laborales, antes que hacer obras de caridad desarticuladas para maquillar los impactos negativos, y muchas veces los costos ambientales y sociales de su actividad.

La nueva época se caracteriza por la desregulación del mundo del trabajo, la contratación unilateral y leonina, la pérdida casi total de garantías para las grandes mayorías y la volatilidad de cualquier estabilidad social y económica. El neocapitalismo destruyó esa sociedad que si bien no era idílica, permitía algunas ventajas. Hoy el mundo del trabajo está invadido por la incertidumbre y no hay ningún sector laboral que pueda decir que está a salvo. Las viviendas, por ejemplo, son objeto voraz de los mercados, los padres y madres deben responder a reglas laborales muchas veces infames y la familia y la escuela han tenido que vivir la transición sin atenuantes. El presente artículo tratará de mostrar la relación entre la violencia social y la crisis del capitalismo global.

\section{Sobre el concepto de violencia y sus dinámicas sociales}

La violencia es ante todo una acción humana con la cual se establece una relación social, una forma de vínculo entre los individuos. Para simplificar las posibilidades, vamos a fundar nuestras reflexiones en una de las más usuales conceptualizaciones: la de la Organización Mundial de la Salud:

El uso intencional de la fuerza o el poder físico, de hecho o como amenaza, contra uno mismo, otra persona o un grupo o comunidad, que cause o tenga muchas probabilidades de causar lesiones, muerte, daños psicológicos, trastornos del desarrollo o privaciones ${ }^{5}$. 


\section{Las violencias microsociales y macrosociales}

En nuestra propuesta, teóricamente las violencias se agrupan en dos grandes campos, de acuerdo a las lógicas de los actores. Las primeras obedecen a estrategias personales y cuando más de pequeños grupos y usualmente son el resultado de situaciones espontáneas y no obedecen a planes o a la acción de estructuras organizadas, o cuando más semiorganizadas, como en el caso de las violencias familiares o comunitarias. A estas las denominamos "violencias microsociales", también conocidas como expresivas o difusas. Otras tienen que ver con estructuras complejas de la sociedad y muchas de ellas se nutren de dinámicas de grandes estructuras, incluso de carácter global, a las que ubicaremos en el campo de las "violencias macrosociales", denominadas también como instrumentales $\mathrm{u}$ organizadas. Allí ubicamos los grandes procesos económicos, políticos y sociales que nutren las grandes violencias organizadas que buscan un determinado fin. Podríamos entonces establecer dos grandes polos en un continuum que nos ayude a comprender la dinámica y naturaleza de las relaciones violentas al interior de la sociedad: en un extremo tendríamos las violencias individuales, por motivaciones puramente espontáneas sin ningún tipo de plan preconcebido, y en el otro extremo tendríamos las violencias altamente organizadas e instrumentadas para determinados fines, por ejemplo las organizaciones de la criminalidad transnacional con sus aparatos de justicia privada, sus redes sicariales, sus mecanismos de toma de decisiones, entre otros, o las organizaciones constituidas para ejercer violencias permanentes con determinados fines políticos, la destrucción del Estado, derrocamiento de un gobierno (entre este tipo de violencias organizadas no se pueden descartar las generadas por los Estados, entre cuyas funciones está el ejercicio de la violencia y la justicia que, dentro de ciertos contextos jurídicos, políticos y sociológicos suelen caracterizarse como "violencia legítima". En otros contextos, cuando no obedece a la legalidad y legitimidad, simplemente "violencia estatal" o en casos extremos "terrorismo de Estado"). Pero entre los dos polos existen muchas modalidades intermedias de acuerdo a las condiciones sociales de cada contexto.

Una dimensión importante para esta caracterización es el grado de complejidad de la organización y la lógica que la mueve: podríamos establecer desde las violencias individuales o espontáneas, por ejemplo, el estallido de ira de uno o varios sujetos, pasando por organizaciones semipermanentes (blandas o móviles) que pueden incurrir en hechos esporádicos de violencia (es el caso de algunas estructuras o formaciones juveniles), hasta las organizaciones 
estables y constituidas con explícitos y permanentes fines donde la acción violenta es uno de sus presupuestos. A estas últimas las denominamos violencias organizadas.

A menudo, las causas de la violencia directa están relacionadas con situaciones de violencia estructural y/o justificadas por la violencia cultural: muchas situaciones son consecuencia de un abuso de poder que cae sobre un grupo oprimido, o de una situación de injusticia social (de un reparto inequitativo de recursos insuficientes, de una gran desigualdad en los ingresos de las personas, dificultad de acceso a los servicios sociales y a la red sanitaria, o la depredación por la fuerza. El caso de la expropiación violenta de la tierra en regiones rurales es notorio en Colombia y en muchos países de América Latina, o el caso de las inequidades étnicas que han invisibilizado y extinguido culturas enteras), y reciben el espaldarazo de discursos que las justifican.

Muchas relaciones y acciones violentas pueden tener de una $y$ de otra. Hay situaciones que se repiten en la vida cotidiana y van creando ambientes de convivencia y tolerancia con la violencia que la "naturalizan" y la banalizan. En épocas de crisis, revueltas y grandes situaciones conflictivas, los dos campos tienden a confundirse. Las conexiones entre unas y otras son el fundamento de fenómenos de expansión de las violencias en varias sociedades del mundo. Cada vez son más frecuentes situaciones difusas, especialmente en ambientes urbanos o de sociedades en situación de conflicto o posconflicto que se tornan inmanejables y ambiguas. Es el caso de las recientes revoluciones árabes: una situación política se agudiza con el uso estatal de la fuerza, hasta el grado de genocidio, mediante bombardeos sobre la población, y termina en violencias sociales, saqueos, violencia sexual y muchas formas de contra violencia.

En América Latina los casos más notorios recientemente fueron las situaciones del posconflicto de las guerras centroamericanas, especialmente de Guatemala y El Salvador, con el surgimiento de las llamadas "maras" o pandillas que aumentaron extraordinariamente su tamaño, convirtiéndose en un fenómeno internacional que ha afectado incluso a ciudades estadounidenses. Cabe resaltar que a partir de 1990 se empezó a abordar el tema de la delincuencia juvenil, tal y como lo señalan las directrices de las Naciones Unidas para la prevención de la delincuencia juvenil, también llamadas Directrices de Riad' .

6 Organización Mundial de la Salud (2003). Informe mundial de violencia y de la salud. p.5, cap. 1 
En Colombia otro caso importante, en términos generales, fue la ciudad de Medellín en los años de mayor violencia, años ochenta y noventa del siglo pasado, cuando a la organización de milicias urbanas por los grupos guerrilleros sobrevino la cooptación de pandillas y grupos juveniles barriales por parte de las organizaciones del narcotráfico, generando un auge del sicariato y la emergencia de grupos autónomos de jóvenes que de la pandilla barrial hicieron rápido tránsito a bandas al servicio de los carteles, llevando a situaciones críticas a toda la sociedad colombiana, paralelamente acompañadas de violencias difusas, riñas callejeras, violencia intrafamiliar, violencia escolar que llevó al incremento de ataques graves contra la integridad de los maestros y directivos docentes por problemas cotidianos de las escuelas ${ }^{7}$. Igualmente, Bogotá en los años 80 creció de manera importante en sus indicadores de violencias difusas, a la sombra del conflicto armado que solo llegó a explicar un máximo del $20 \%$ de los hechos de sangre. Tal vez esta correlación en el aumento de los hechos violentos tenga que ver con el aumento de la circulación de armas de fuego. Durante varios años cerca del $80 \%$ de los homicidios de Colombia fueron ocasionados con

Corporación Región (1990). Violencia juvenil. Diagnóstico y alternativas. Medellín: Corporación Región. P. 20-30. Disponible en internet: http://www.region.org.co/index.php/publicaciones/cat_view/44-libros/66-1990. Consultado 10/09/2011 armas de fuego. Está comprobado que la posesión de una arma de fuego incrementa 2.7 veces el riesgo de muerte de las personas próximas a quien la posee. $\mathrm{Y}$ en momentos de crisis social y de incremento de la criminalidad se potencian los riesgos al circular las armas en los grupos delincuenciales.

Pero no se necesitan grandes crisis o circunstancias extraordinarias. Las situaciones endémicas de las ciudades latinoamericanas hacen cada vez más compleja y borrosa la frontera entre las violencias macro y microsociales. La expansión de la pobreza, la informalidad y las economías ilegales, han creado climas propicios para que esas fronteras sean cada vez más difusas. En el caso de Bogotá, por ejemplo, la correlación entre uno y otro indicador es del 94\%. Las localidades en donde se presenta una alta incidencia de homicidios instrumentales son precisamente aquellas donde se registra un mayor número de casos impulsivos, especialmente de muertes por riña ${ }^{8}$. En otras palabras las diferentes formas de violencia cada vez más se conectan y se retroalimentan.

\section{Jóvenes y relaciones de violencia}

En la coyuntura actual de la sociedad colombiana son cada vez más

8 Llorente, M. V. y otros (2000). Violencia homici da y estructuras criminales en Bogotá. Bogotá: Universidad de los Andes. p.14. 
frecuentes y notorias las violencias en las que participan los jóvenes en diversos escenarios y situaciones. Aunque hay que advertir que existen estereotipos prejuiciados que buscan presentar como violentos o delincuenciales los grupos juveniles de toda índole. Hecha esta salvedad, es innegable que a nivel global se está dando el fenómeno del involucramiento cada vez mayor de sectores de la población joven en situaciones violentas que la hacen un sector altamente vulnerable. Una hipótesis ronda este trabajo: la conexión más eficiente en la sociedad contemporánea entre las violencias macro y micro son las múltiples expresiones de violencia juvenil, dada por sus estructuras móviles y semiorganizadas que fácilmente derivan en situaciones permanentes o esporádicas de delincuencia que las hace proclives a verse involucradas en circunstancias violentas. Lo que a su vez las coloca en la riesgosa frontera de las violencias organizadas. Ello obedece a dinámicas sociales, económicas y culturales muchas veces globales, otras veces regionales y locales que pasaremos a explicar y que han sido abordadas por diferentes estudios como los aquí referenciados. La temática sobre juventud tiene nuevos desarrollos en los estudios sociales, como el de las culturas juveniles ${ }^{9}$, la autonomización de las pandillas barriales ${ }^{10}$ y los grupos de pares que se forman y emergen de las escuelas proyectándose sobre las comunidades barriales ${ }^{11}$. Son situaciones emparentadas pero diferenciables.

\section{De la informalidad y la ilegalidad}

La informalidad en las economías es señalada como uno de los grandes temas del que se derivan innumerables problemas de las sociedades de los países latinoamericanos. Colombia no se exime de ellos. Un reciente estudio caracteriza así a los informales:

Los informales son principalmente individuos de bajos ingresos, trabajan mayoritariamente en establecimientos de menor tamaño y viven en ciudades periféricas. En cuanto al género, son más propensas a estar en la informalidad las mujeres que los hombres. Asimismo, el

9 Reguillo Cruz, R. (2000). Emergencia de las culturas juveniles. Estrategias del desencanto. Bogotá: Grupo Editorial Norma.

10 Perea Restrepo, C. (2007). Con el diablo adentro: pandillas, tiempo paralelo y poder. México: Siglo XXI.

11 Ruiz Botero, L. (2006). La escuela: territorio de frontera. Tipología de conflictos escolares según estudio comparado en Bogotá, Cali, Medellín. Medellín: Instituto Popular de Capacitación. 
perfil de la informalidad está caracterizado por ser empleados de bajo nivel educativo, en su mayoría jóvenes. Por otro lado, son más propensos a estar en el sector informal las categorías ocupacionales de cuenta propia y los trabajadores familiares $^{12}$.

Para el año 2012, el Banco de la República estima que el $62 \%$ de los trabajadores colombianos están en la informalidad y que no más de cuatro de cada diez trabajadores gozan de un puesto formal, lo que condena a los otros seis a un ingreso inferior. El 88\% de los trabajadores no recibe primas; el $67 \%$ no tiene vacaciones con sueldo y el $62 \%$ no tiene asegurada una pensión ni servicio de salud a no ser que haya sido afiliado al servicio del Sisben o sistema estatal para la población de más bajos recursos.

La informalidad abre los tránsitos hacia las ilegalidades y hacia las si-

12 Galvis, L. (2012). Informalidad laboral en las áreas urbanas de Colombia. Cartagena: Banco de la República. Febrero 2012.http://www. banrep.gov.co/documentos/publicaciones/ regional/documentos/DTSER-164.pdf Visita de 19-03-2012.http://www.eltiempo.com/ economia/finanzas-personales/ARTICULO-WEBNEW_NOTA_INTERIOR-11376243.html Visita de 19-03-2012 tuaciones de violencia ${ }^{13}$. Con altísima frecuencia la población latinoamericana tiene que sobrevivir del denominado "rebusque", nomadismo laboral informal y de desempleo disfrazado que impide a las familias tener un proyecto de vida estable, $y$ donde los jóvenes Ilevan la peor parte. Estas circunstancias han hecho que los niños y niñas de los sectores más pobres acompañen a los padres $y$ hermanos en actividades callejeras donde se dan aprendizajes indeseables que causan un fácil paso de la informalidad a la ilegalidad.

Estos aprendizajes fácilmente se transmiten en la escuela. Los estudios recientes en Bogotá revelan que el hurto es la infracción predominante en los ámbitos educativos. Desde los primeros años se pasa del hurto oportunista, esporádico y casual, al hurto frecuente e intencionado que vincula al infante o adolescente con el mercado ilegal de objetos robados. Esta actividad es el puente para que el niño, niña o joven se vincule a sitios de venta de artículos usados. La actividad más frecuente era el hurto de ciertos textos escolares que tenían mercado asegurado en las librerías de usados que se ubicaban en el centro de la

\footnotetext{
13 Lemus Murcia, O. (2007) Identificación, análisis y propuestas de políticas públicas para la prevención y control de las acciones de los ciudadanos que se desarrollan en torno al tránsito de la legalidad a la ilegalidad y viceversa en Bogotá Bogotá: Secretaría de Gobierno de Bogotá D.C., Observatorio de Convivencia y Seguridad Ciudadana, SUIVD Sistema Unificado de Información de Violencia y Delincuencia. En línea: www. ceacsc.gov.co/index.
} 
ciudad, luego se popularizó el mercado de otros objetos de valor como relojes, plumas o lapiceros de lujo, implementos de mayor valor como calculadoras electrónicas. Hoy, el mercado ilegal más usual es el de teléfonos móviles o celulares, artefactos digitales y computadores portátiles. Pero también, a nivel de la ciudad, el mayor indicador de inseguridad urbana es el hurto a residencias y así lo reflejan las investigaciones adelantadas por el Observatorio de Convivencia y Seguridad Ciudadana en los últimos años ${ }^{14}$, es decir que podría establecerse un puente real entre el hurto pequeño que se inicia en la casa, se amplifica en la escuela y luego se conecta con las redes delincuenciales que operan a nivel urbano. De hecho, en algunas instituciones educativas que participaron en la investigación arriba señalada, las familias participantes pudieron visibilizar las interconexiones del hurto en la casa, la escuela, el barrio y la ciudad analizando experiencias de sus hijos escolarizados.

En el marco de este clima urbano general se insertan las organizaciones o estructuras de pares que se articulan en la escuela pero interactúan en el barrio o vecindario y en la ciudad, que cuando se autonomizan y para mantener sus consumos, usan el pequeño hurto como financiador de su autonomía en el mundo de los adultos. Así, en las barriadas las mencionadas organizaciones de jóvenes o adolescentes se pueden transformar en "parches" o pandillas que hacen el tránsito a pequeñas estructuras "semiorganizadas" o "blandas" que se mueven en la frontera de la legalidad, o se transforman en organizaciones en "conflicto con la ley", lo que las hace fácilmente instrumentables por organizaciones "para el crimen" o por estructuras "duras" y permanentes del crimen organizado, como hemos dicho, iniciando el camino del deterioro social y de conflicto con la escuela, la familia y la propia comunidad barrial.

A este conjunto de fenómenos se le suma el deterioro creciente de la ciudad latinoamericana y mundial:

La nueva miseria urbana, un fenómeno surgido a cabaIlo de la incesante polarización económica desatada al interior de las ciudades. Desarrollado durante las últimas décadas del siglo $\mathrm{XX}$, este proceso dio origen a un paisaje urbano en el que conviven, separadas a veces por

14 Álvarez, C. (2007). Hurto a residencias en Bogotá 1999-2006 - Estudio exploratorio. Bogotá: Secretaría de Gobierno de Bogotá D.C., Observatorio de Convivencia y Seguridad Ciudadana, SUIVD Sistema Unificado de Información de Violencia y Delincuencia. Ubicado en: www.ceacsc.gov.co/index. Consultado en octubre 1 de 2011. 
pocos metros, la mayor de

las opulencias burguesas junto a la miseria más estremecedora $^{15}$.

Esto ha dado lugar a lo que Manuel Castells ha denominado "ciudades duales", donde coexisten zonas de "riqueza impúdica" que lindan con extensas zonas de miseria, en donde los jóvenes llevan la peor parte de las formas del capitalismo posmoderno: acceso restringido a la educación de calidad, especialmente a la formación superior, ausencia de vínculo con la economía formal, precarización del contrato laboral, inestabilidad, ausencia de seguridad social, deterioro creciente de los salarios. El peor mundo posible, el mundo de la desesperanza, que la sociedad tiene que pagar en términos de inseguridad, banalización de la violencia, criminalización de la barriada ${ }^{16}$.

Esta realidad se puede resumir en lo que Pierre Bourdieu denominó como la "ley de la conservación de la violencia":

No se puede jugar con la ley de la conservación de la violencia: toda la violencia se paga $y$, por

15 Mozzo, E. (2001). Capitalismo hoy: La nueva marginalidad. Acerca del libro Parias Urbanos. Marginalidad en la ciudad a comienzos del milenio, de Loic Wacquant. En: Revista Herramienta. No. 16- http://www.herramienta.com.ar/revista-herramienta-n-16/capitalismo-hoy-la-nueva-marginalidad-acerca-del-libro-parias-de-la-ciudadm Consultado 05-01-2011

16 Castells, M. (1995). La ciudad informacional. Madrid: Alianza Editorial. Además: Sassen, Saskia (2000). Cities in a world economy. Londres: Pine Forge Press. ejemplo, la violencia estructural ejercida por los mercados financieros, en las formas de despidos, pérdida de seguridad, etcétera, es equiparada, más tarde o más temprano, en forma de suicidios, crimen y delincuencia, adicción a las drogas, alcoholismo, un sinnúmero de pequeños y grandes actos de violencia cotidiana ${ }^{17}$.

\section{Años 90 y crisis social}

En la década de los 90, el sistema educativo latinoamericano es impactado por procesos simultáneos: la llegada de numerosos niños y jóvenes a las escuelas básicas, debido a esfuerzos sostenidos por los gobiernos de ampliación de cobertura educativa con base en políticas de los organismos multilaterales, y por las políticas de flexibilización laboral,simultáneamente con las posiciones más radicales sobre la disminución de la intervención estatal en las economías de mercado (neoliberalismo), que aumentaron sensiblemente el desempleo, hicieron precario el contrato laboral, aumentaron la desocupación femenina y juvenil afianzando la situación de inestabilidad social. Por último, el Ilamado "bono demográfico" consistente en la disminución de la población de niños en educación 
primaria lo que permitió ampliar la cobertura de la educación secundaría ${ }^{18}$.

Después de 20 años de hegemonía neoliberal (1989-2009), la sociedad se ve enfrentada al gran desajuste social que produjo la sostenida reforma laboral, la informalización del empleo y la disminución de las garantías sociales (subsidios, programas de bienestar, entre otros), especialmente la disminución del acceso a la seguridad social y a la vivienda digna, siendo la juventud la población más afectada por el deterioro social a nivel mundial.

La incertidumbre ocupacional, las nuevas configuraciones familiares, el ausentismo escolar, el aumento de consumos de alcohol y psicoactivos y el aumento de la violencia social, han sido los principales efectos.

En el informe del 2008, la Cepal establece que en América Latina las muertes de jóvenes se deben a causas externas, homicidios, suicidios, accidentes (141 por cada 100.000 habitantes). Colombia ocupa el segundo lugar después de Venezuela con 81 muertes por 100.000 habitantes $^{19}$.

\section{La revolución informática}

Pero el auge de las políticas neoliberales no es la única causa global: los cambios introducidos por la revolución informática y digital son la otra gran causa global que transformó el mundo del trabajo, especialmente a partir de los años 90 y acelerada de manera formidable a partir de 1996, con la difusión de Internet, que junto con la consolidación de la llamada sociedad del conocimiento $^{20}$, han producido la más formidable revolución laboral desde la primera revolución industrial. Junto con las políticas proteccionistas del primer mundo y las políticas antimigratorias, han hecho que la reconversión industrial enriquezca a las grandes corporaciones del primer mundo y empobrezca, flexibilice y vuelva precario y vulnerable el empleo en el tercer mundo,

18 Behrman J., Duryea, S. y Székely M. (2003) Aging and Economic Opportunities: What Can Latin America Learn from the Rest of the World? En: The Family in Flux: Household Decision-Making in Latin America. Washington: IDB.

19 Cepal, Panorama Social de América Latina, 2008, apoyada por fuentes de la Organización Panamericana de la Salud, OPS, Estadísticas de la salud en las Américas. Ediciones 2003 y 2006. Capítulo IV. p. 174-175

20 En términos de ciencia y tecnología se afirma que la sociedad del siglo XXI es la sociedad de la información y de aquí se ha pasado a la sociedad del conocimiento, lo cua implica un cambio al énfasis del valor agregado: valen más los productos virtuales e intangibles que condensan información y conocimiento, que los bienes tangibles de mercado. Drucker, P. (1969). La era de la discontinuidad. Londres: William Heinemann Ltd. Drucker, P. (1993). La sociedad poscapitalista. Barcelona: Apóstrofe. 
generando migraciones masivas, muchas de ellas ilegales, hacia el primer mundo, especialmente de jóvenes desempleados, propiciando el tráfico de personas como uno de los grandes negocios de las mafias, ligado a la prostitución, la desintegración familiar y formas de esclavitud laboral de los migrantes ilegales. Manuel Castells va más allá y plantea un cambio sustancial en el capitalismo industrial hasta ahora conocido y con importantes argumentos, afirmando cambios fundamentales en el modo de producción e incluso el surgimiento de uno nuevo que se ha denominado modo de producción informacional ${ }^{21}$.

Para el caso colombiano se calcula que cerca de 6 millones de colombianos se encuentran en el exterior por diferentes motivos ${ }^{22}$ y hasta mayo de 2011 el Gobierno registró más de 3,7 millones de desplazados internos en el país. La ONG Consultoría para los Derechos Humanos y el Desplazamiento (Codhes), considera que la cifra real de desplazados por el conflicto armado interno desde mediados de los años 80 supera los 5 millones de personas ${ }^{23}$. Todo

21 Castells, M. (2006). La era de la información: economía, sociedad y cultura. Mexico: Siglo XXI Editores. Quinta Edición. Vol. III Pg.95-195.

22 Hernandez, M. En 2010, la cifra de colombianos residentes en el exterior llegará a los seis millones. Comunicado de prensa. Colombianos en Nueva York. 5 de enero de 2010. En línea: http://www.conexioncolombia.com/Comunicado-enero5-2010.pdf. P. 1. Consultado el 20 de septiembre de 2011.

23 Acnur. Desplazamiento Interno en Colombia. En línea. Consultado el 19 de septiembre de 2011. Disponible en: http://www.acnur.org/t3/ operaciones/situacion-colombia/lineas-estrategicas-y-objetivos-del-acnur/ este conjunto de fenómenos han sido denominados como "sociedad posindustrial", "capitalismo posmoderno", "neocapitalismo" o "sociedad informacional" 24 .

\section{Contra-revolución social a partir del mundo del trabajo}

Ya no hay duda: en el neocapitalismo hay una nueva división del trabajo y un nuevo concepto del trabajo flexible:

Lo nuevo en realidad es la ductilidad, el trabajo flexible, trayectorias con empleos versátiles, no repetitivos, pero sin tradición y sin historia. Las relaciones interpersonales que ha fabricado el neocapitalismo son evanescentes, constituidas con base en fragmentos. Los hijos no repiten la rutina de sus padres.Por tanto, el carácter de sus padres no es su carácter ${ }^{25}$.

Los rasgos que señaló Richard Sennett, uno de los estudiosos del nuevo capitalismo, se definen así:

Lo que hoy tiene de particular la incertidumbre es que existe sin la amenaza de un desastre histórico, y en cambio está

24 Castells, M. Op.cit. P. 56.

25 Kaplan, C. (2006). La inclusión como posibilidad. Buenos Aires: Ministerio de Educación Ciencia y Tecnología. Organización de los Estados Americanos. Agencia Internacional para la Cooperación y el Desarrollo. P. 22. 
integrada en las prácticas cotidianas de un capitalismo vigoroso... La consigna "nada a largo plazo desorienta la acción planificada, disuelve los vínculos de confianza y compromiso y separa la voluntad del comportamiento ${ }^{26}$.

La incertidumbre, integrada al riesgo como condición, ha introducido cambios en la mentalidad de todos los seres humanos que se mueven al ritmo de los nuevos mandatos, de la nueva sociedad dominante, que introduce retóricos vertiginosos, donde los jóvenes y los niños fueron los primeros que aprendieron.

La cultura moderna del riesgo se caracteriza porque no moverse es sinónimo de fracaso, y la estabilidad parece casi una muerte en vida. Por lo tanto, el destino importa menos que el acto de partir. Inmensas fuerzas económicas y sociales dan forma a la insistencia de marcharse; el desorden de las instituciones, el sistema de producción flexible, realidades materiales que se hacen a la mar. Quedarse quieto equivale a quedarse fuera del juego ${ }^{27}$.

La consigna "nada a largo plazo" ha sido interiorizada especialmente por la juventud que atraviesa el tiempo del "no lugar" y el "no futuro". Los padres y madres de familia cambian de lugar de trabajo, de pareja, de barrio, de ciudad y muchas veces de país. Deben cambiar con frecuencia de empresa, de oficios, e incluso de profesión. Al respecto, Manuel Castells plantea que en promedio actualmente se puede cambiar de profesión siete veces en la vida y según Sennett, un joven norteamericano puede cambiar 11 veces la vida laboral ${ }^{28}$, lo cual constituye prácticamente la incapacidad de realizar una "carrera entendida como ruta vital" ${ }^{29}$, el término cuyo significado original era el camino para corregirse, aplicado posteriormente al trabajo, designa el canal por donde se encausan las actividades profesionales de toda una vida

El capitalismo flexible ha bloqueado el camino recto de la carrera, desviando a los empleados repentinamente de un tipo de trabajo a otro. En inglés del siglo XVI, la palabra job (trabajo, empleo) designaba un pedazo o fragmento de algo que podía acarrearse.

26 Sennett, R. (2000). La corrosión del carácter. Las consecuencias personales del trabajo en el nuevo capitalismo. Barcelona: Anagrama. P. 30-31.

27 Sennett, R. Op. Cit. p.91.

28 Sennett, R. Op. Cit. p.20

29 Roig-lbañez J. (2006). La educación ante un nuevo orden mundial. Buenos Aires: Díaz de Santos Ediciones. P. 250 
Hoy la flexibilidad le devuelve ese sentido desconocido, pues a lo largo de su vida la gente hace fragmentos de trabajo ${ }^{30}$.

\section{Exclusión, pobreza, discriminación, informalidad}

Uno de los estudios recientes sobre el tema de la ciudad latinoamericana contemporánea ubica los siguientes problemas de carácter estructural que ayudan a explicar los fenómenos violentos ${ }^{31}$ :

En primer lugar, la exclusión social, la pobreza, la discriminación y la informalidad; según la Organización Internacional del Trabajo, casi uno de cada dos trabajadores urbanos latinoamericanos son informales y constituyen un terreno fértil para las actividades ilícitas. De igual manera, la concentración socioespacial de la pobreza y la informalidad, el fenómeno de la "ciudad dividida", está muy relacionado con la violencia urbana.

La ausencia de "inserción estable" en el mundo laboral crea las condiciones para la trashumancia, que hace vulnerables a todas las personas pero especialmente a los

30 Sennett, R. Op. Cit. p.21.

31 Mesa, M. Violencia social y globalización en América Latina. Op. cit. jóvenes. El deseo de cambiar su condición y ascender socialmente permite que muchos, hombres y mujeres, pierdan la paciencia sobre los mecanismos tradicionales para "surgir". También influye.

La debilidad de las instituciones, que se traduce en vacíos locales de gobierno. En las barriadas y favelas de las grandes ciudades latinoamericanas, estos vacíos se forman a raíz de una prolongada ausencia de las autoridades y de los representantes de la ley. A menudo, los actores armados privados e informales ocupan el lugar de la policía y la justicia... las bandas armadas la imponen por la fuerza, pues es un requisito para poder desarrollar su actividad delictiva, generalmente asociada al narcotráfico ${ }^{32}$.

Las bandas, las pandillas y las maras encontraron un territorio fácil de dominar ante la ausencia de agentes estatales. De otra parte, las redes del microtráfico y cobro de vacunas y extorsiones se convirtieron en fácil mecanismo para no depender de la economía del trabajo. Por el contrario, muchos jóvenes ahora tendrán poder y serán importantes, ganándose un lugar que la ciudad les negó. 
La exclusión, la discriminación, son la otra cara de los círculos viciosos de la pobreza y la violencia. Los jóvenes de los barrios más pobres conviven cotidianamente con los riesgos de violencia, drogadicción, promiscuidad, prostitución, atraco y su inserción laboral exitosa es menos probable, dado que en estas circunstancias la deserción escolar es frecuente y si logra terminar la secundaria es difícil que alcance la educación superior, debido a las bajas tasas de cobertura en Colombia. Aunque los indicadores oficiales para 2002 hablaban de aproximadamente $16 \%$ de educación universitaria, desde ese año el gobierno nacional incluyó en las cifras de educación superior los datos del Servicio Nacional de Aprendizaje, SENA, de formación para el trabajo, que elevó automáticamente las cifras al 24,5\%, para ese año, y estimando la cobertura para el año 2010 en 37\%33. Aún con esta maroma estadística, es una baja cobertura comparativamente con América Latina,lo que significa que de cada 100 bachilleres 63 no acceden a ningún tipo de educación superior ${ }^{34}$.

\section{Las herencias de la guerra}

Otro factor importante que afrontan las sociedades latinoamericanas que han tenido conflictos armados son las herencias de la guerra, cuyas consecuencias se expanden por el vecindario. El caso colombiano es paradigmático en la medida que alberga uno de los dos conflictos armados más antiguos del planeta, lejos de encontrar solución.

Las prolongadas guerras civiles de los países centroamericanos y andinos han dejado un legado de violencia y criminalidad y un gran número de armas de guerra ahora utilizadas para actividades delictivas. Los actuales responsables de la violencia son en muchos casos actores no estatales que operan en enclaves urbanos pobres $y$ marginales, en ocasiones surgidos de procesos de desmovilización de las fuerzas armadas, los paramilitares

33 Recientemente fue publicado un informe que puede ser la clave de uno de los casos de impunidad más graves de toda la historia de América: "Memoria de la impunidad en Antioquia", donde el IPC y la Corporación Jurídica Libertad decidieron recopilar "lo que la justicia no quiso ver frente al paramilitarismo". De otra parte, la Corporación Nuevo Arco Iris ha publicado informes sobre varias regiones que han comprometido los procesos electorales de muchos dignatarios electos por voto popular demostrando su relación con grupos armados.

34 Ver: http://www.dnp.gov.co/Programas/Educaci\%C3\%B3nyculturasaludempleoypobre za/Subdirecci\%C3\%B3ndeEducaci\%C3\%B3n/Educaci\%C3\%B3nSuperior.aspx Visitado 19-03-2012 
y los grupos guerrilleros. Empero, también emergen bandas criminales que han logrado tener el monopolio de la violencia...en países como Colombia, Guatemala y México y en un sentido más restringido, en Argentina, Brasil, El Salvador, Honduras y Perú ${ }^{35}$.

A juzgar por las consecuencias, la reinserción sin prevención es más riesgosa que la misma guerra, máxime, como en el caso colombiano, cuando la guerra no ha terminado. $O$ dicho de otra manera: una paz mal hecha es tan mala como la guerra misma. Las posguerras son un camino de grandes dificultades donde se ve que las secuelas de la guerra no terminan cuando llega la paz. La elevación de la delincuencia urbana en Colombia, el rearme de la Ilamadas "bandas emergentes", la reconstitución de los ejércitos narcoparamilitares, aunque ya no con la misma fuerza, es una realidad con la que la ciudad colombiana tiene que convivir. En el caso de Bogotá, algunos de esos desmovilizados se han transformado en delincuentes profesionales que están deteriorando aceleradamente la vida ciudadana.

La corrupción se convierte en otro factor influyente. En muchos casos las mafias y las organizaciones criminales han tenido o tienen acceso al gobierno. $\mathrm{Y}$ este, lamentablemente, es el escenario más negativo en muchos países latinoamericanos. Economías ilegales han Ilegado a controlar el poder local y regional. El caso colombiano, que no es diferente al de otros países, hasta ahora asoma su cara oscura. Cerca del $30 \%$ del parlamento ha tenido algún tipo de proceso penal o disciplinario por vínculos con organizaciones de autodefensa financiadas por las mafias. En varias regiones las instituciones locales fueron copadas por poderes mafiosos e incluso hubo zonas donde varios grupos de guerrillas o paramilitares se enfrentaron en pequeñas guerras por los recursos del Estado.

Otro de los factores que se anotan es la situación generalizada de impunidad. El sistema carcelario es totalmente inefectivo en sus fines de rehabilitación y es sabido que las cárceles son escuelas del delito y oficinas para la comisión de nuevos delitos desde la misma cárcel. Pero lo más sorprendente son los tiempos de duración de los procesos y que más del $50 \%$ de los reos están en calidad de detención previa al juicio. Los índices de impunidad y subregistro de los homicidios son similares en toda la región. México, Brasil, Guatemala 96\%, Colombia 97\%. En términos generales, la probabilidad de condena por un delito en Colombia no supera el 20\%. En materia de homicidio tal probabilidad gira en torno al $3 \% .^{36}$

\footnotetext{
36 Rivera, S. y Barreto, L. La impunidad en el sistema penal acusatorio en Colombia. Bogotá: Ministerio del Interior y Justicia. Documento de trabajo, s.f. www.mij.gov.co/econtent/library/.../ DocNewsNo4362DocumentNo2463.PDF. Consultado 07-01-2011
} 


\section{El prohibicionismo que distorsiona todo}

A lo anterior se suma el prohibicionismo mundial de la llamada guerra contra las drogas, que distorsiona los precios y proporciona grandes ganancias a las mafias asociadas al narcotráfico ${ }^{37}$. Además, trastoca prioridades. La presión internacional para obtener ayuda exige resultados. Perú, Colombia y ahora México, han recibido ayuda estadounidense para la guerra contra las drogas, que dicho sea de paso es uno de los fracasos más rotundos de la política internacional de los últimos años, con efectos perversos múltiples. En el caso colombiano es evidente: en la lucha contra el "narcoterrorismo" se pasó de gasto en la guerra de 2 $\%$ del PIB en el 2002 a $3.8 \%$ en el 2010. Dicha guerra solo explica del $5 \%$ al $20 \%$ de las muertes violentas al año. En tanto la criminalidad y las violencias urbanas, que producen el más alto porcentaje de homicidios, no reciben la atención ni la inversión social necesaria para contrarrestarla.

La política criminal de estos países equivocadamente ha puesto el foco de manera exagerada en la persecución a los delitos asociados, con un evidente desgaste, y cuando el fenómeno se reduce en un país, simplemente migra a otro. $\mathrm{Si}$ se derrotan los grandes carteles, como sucedió en los años 90 en Colombia, aparecen microcarteles y los negocios transnacionales no disminuyen el flujo de cocaína hacia los Estados Unidos o Europa, sino que, por el contrario, el consumo ha aumentado sustancialmente. En tanto que el prohibicionismo causa efectos perversos en los precios ayudando a mantener exorbitantes tasas de ganancias.

\section{Violencia urbana}

Es el conjunto de manifestaciones violentas que se dan en espacios urbanos. Y aunque no hay una definición específica, todos los estudios hablan de una violencia especial que se desarroIla en las ciudades, de una alta concentración de delitos y de una permanente sensación de inseguridad de los ciudadanos. El ensayista mexicano Carlos Monsiváis, sin ser un analista social, más un escritor de crítica cultural, la caracterizó a así:

37 Es abundante la literatura al respecto. Una argumentación desde la economía política está en Guerrero, Javier (1996). Los antecedentes internacionales de la crisis colombiana. En: Pensamiento y acción. Colombia, UPTC, vol. 1: págs: 7 - 14 y Guerrero, Javier (2002) Mercados de violencia y guerra civil en América Latina en los 90. En: Apuntes del Cenes. UPTC, vol. 22 págs: $263-293$. 
Los conflictos, las tragedias, las conductas límite propiciadas por la crisis del Estado de derecho, el perpetuo estallido -económico, social y demográfico- de las ciudades, y la imposibilidad de una efectiva seguridad pública, sea por la ineficiencia de los cuerpos encargados o por la "feudalización" imperante en barrios y colonias. Violencia urbana es el amplio espectro de situaciones delincuenciales, ejercicios de supremacía machista, ignorancia y desprecio de los derechos humanos, tradiciones de indiferencia aterrada ante los desmanes, anarquía salvaje y desconocimiento de la norma ${ }^{38}$.

Si miramos con detenimiento esta definición vemos que allí cabe todo tipo de hechos violentos y delincuenciales. Por eso la violencia urbana ha sido ante todo un concepto de la criminología y la sociología más que de otras disciplinas. Pero ¿qué caracteriza a la ciudad contemporánea para que se haga tanto énfasis en las violencias urbanas? Una de las características más notorias de la

38 Monsiváis, C. (1999). Notas sobre violencia urbana. Revista Letras Libres. [En línea], Mayo 1999, No. 5 [Consultado 25, septiembre, 2010] p. 34-39. Disponible en: < http://www.letraslibres.com/index.php?art=5795>. ciudad es que por ser la principal aglomeración de la vida, se convierte en la mayor concentración de la muerte de variadas formas. Desde los accidentes de tránsito hasta los homicidios y suicidios de las más variadas formas. La otra característica es la participación de los jóvenes en hechos de violencia. Pero es una consecuencia lógica de los porcentajes de población entre los 15 y los 25 años, la forma como ellos se apropian del espacio urbano, la intensidad de las relaciones sociales, hacen que sean los que más interactúan por la ciudad y los que frecuentan horarios y situaciones de riesgo, lo que se tiene que reflejar estadísticamente $^{39}$. No olvidemos que la violencia es ante todo consecuencia de unas relaciones sociales en contextos específicos.

Además de estas características intrínsecas de las sociedades urbanas, la ciudad latinoamericana tiene unas características que la hacen distinta a las ciudades del primer mundo: 1 . receptoras de múltiples actores de los diferentes conflictos armados, 2. la exclusión social, la pobreza y la discriminación, 3. la informalidadilegalidad, 4. la ineficacia y la corrupción.

39 Al respecto se pueden revisar las estadísticas del INML-CF de los últimos 10 años, en donde se registra que la población de mayor riesgo social que sufre situaciones de violencia como lesiones personales, maltrato infantil y violencia escolar, es la población entre 10 y 17 años, es decir, en su gran mayoría población escolarizada. 


\section{Urbanización conflictiva}

Después del final de la guerra fría los conflictos de América Latina cambiaron. Ya no son las guerrillas rurales y urbanas el fenómeno más notorio. Las guerrillas, que las hubo en casi todos los países de la región después del triunfo de la revolución cubana, desaparecieron gradualmente, algunas derrotadas como en los países que sucumbieron a las dictaduras, otras que negociaron procesos de paz, y solamente dos casos que lograron el triunfo de sus respectivas revoluciones: Cuba y Nicaragua. El caso colombiano es sui generis, en la medida que aún sobrevive una de las dos guerras más antiguas del planeta, y la única en el hemisferio occidental donde predomina un proceso de insurgencia crónica sin revolución. Esto además se sumó al convergente fenómeno de los años ochenta cuando emergió el narcotráfico con sus poderosos carteles. No obstante que muchos de esos fenómenos cesaron o mutaron en su intensidad, las secuelas siguen ahí:

Es evidente que en América Latina la violencia políticomilitar ha disminuido, pero se han agravado otros tipos de violencia social y el crimen organizado en redes transnacionales. Los homicidios dolosos alcanzan 25,1 por cada 100.000 habitantes, el nivel más alto del mundo y tres veces la media mundial.... En las dos últimas décadas, la violencia fue la principal causa de muerte entre los latinoamericanos de entre 15 y 44 años. El coste económico de la violencia alcanza niveles muy altos. El Banco Interamericano de Desarrollo estima que este se situaría entre los 140.000 y los 170.000 millones de dólares anuales, lo que equivale al $12 \%$ del PIB anual regional.... Buena parte de esos costes es atribuible a la contratación de seguridad privada, solo accesible a los más ricos, lo que supone un factor añadido de desigualdad en la región ${ }^{40}$.

Pero ¿qué es lo que hace que la ciudad latinoamericana sea tan conflictiva? Muchos factores pueden explicar estas circunstancias. La primera es la fractura social: las diferencias sociales son abismales. Las brechas entre ricos y pobres evidentes. La ciudad latinoamericana tiene rota su geografía, más que cualquier tipo de ciudad de otras regiones del mundo, en zonas de miseria, zo- 
nas de clases medias y zonas exclusivas para muy ricos. América Latina es una región con grandes desigualdades; desigual no solo económicamente sino en el acceso a las instituciones, a los bienes y a los servicios. Otros factores estarían asociados a la inacción y debilidad institucional, que se suman a la ineficacia y la corupción, que llevan a los cuerpos policiales a competir con poderosas organizaciones criminales que permanentemente amedrentan e interfieren la acción de la justicia. Otro de los problemas es el legado de los conflictos armados de las últimas décadas. Al final de las guerras centroamericanas emergieron las Ilamadas "maras", grandes pandillas juveniles de tamaño y capacidad de acción nunca vista, globalizadas en la medida en que se mueven en varios países, como los Estados Unidos, por ejemplo.

En el caso colombiano, aunque el conflicto armado disminuyó en su intensidad, un imperfecto proceso de desmovilización ha hecho que en las principales ciudades se alojen 53.659 desmovilizados de la lucha armada. Se calcula que al menos 30 mil de ellos se encuentran en la ciudad de Bogotá, donde la criminalidad organizada ha crecido significativamente en los últimos dos años ${ }^{41}$.

41 Especialmente de las Autodefensas Unidas de Colombia, especie de ejército de contrainsurgencia de los narcotraficantes que negoció su desmovilización con el gobierno mediante los mecanismos de la Ley de Justicia y Paz (Ley 975 de 2005).
Pero todas estas manifestaciones violentas son la parte visible de los fenómenos. El caso colombiano es muy diciente: tiene el $49 \%$ de su población en situación de pobreza y el $14 \%$ en condiciones de indigencia. Presenta tasas de desempleo crónico de dos dígitos. 2,5 millones de personas mayores de 15 años analfabetas, 750 mil niñas y niños entre 5 y 11 años por fuera del sistema educativo de preescolar y primaria, 1,2 millones de adolescentes entre 12 y 17 años desescolarizados, 3,9 millones de jóvenes entre 18 y 24 años aún no asisten a la educación superior o de formación para el trabajo. Un alto porcentaje de esos niños y jóvenes viven en las barriadas de las ciudades capitales. Estructuralmente estas personas están por fuera de toda posibilidad de vida digna.

\section{Conflictos barriales asociados a dinámicas escolares y juveniles}

Así como la metrópoli es un fenómeno macrosocial, el barrio es su correspondiente fenómeno micro. La ciudad de la cual hablamos en el caso de los jóvenes no es la de los grandes centros de negocios ni la de las grandes zonas comerciales. La realidad social que nos interesa para analizar las relaciones de violencia y jóvenes es la vida de la barriada donde transcurre la mayoría de su tiempo. En las ciudades tradicionales y antes de las metrópolis los barrios 
tenían sus propias escuelas a escala de sus necesidades. A medida que la ciudad se desarrolló, obviamente el tamaño de aquellas creció y hoy se habla de "megacolegios", instituciones de 3000 a 5000 estudiantes, aplicando economías de escala a la población infantil y juvenil. En casi todas estas instituciones se hacen desplazamientos de población estudiantil desde otras zonas de la ciudad o desde otros barrios para cumplir con los cupos asignados. ¿Habrán pensado los planificadores, en los efectos de los que significan estas concentraciones de población en edad escolar para un pequeño vecindario? Además, niños de corta edad deben hacer recorridos a veces de largas horas de ida y regreso, por la ciudad, disminuyendo su tiempo creativo.

Uno de los hallazgos de nuestro estudio muestra cómo en todas las localidades estudiadas, pero especialmente en Fontibón, una pequeña población de origen precolombino que se anexó a la metrópoli y que aún conserva muchos rasgos pueblerinos ${ }^{42}$, la instauración de estas megaestructuras educativas convierte automáticamente al barrio que lo aloja en escenario de conflictos. En esta localidad, donde se concentraron varias instituciones de gran tamaño, se detectaron problemas de diversa índole alrededor de los mismas: ventas ambulantes de drogas, atracos de los jóvenes a los niños, enfrentamientos entre combos, pandillas y barras, "chiquitecas" 43, "bares de segundo piso". Pero lo más destacado fue la rivalidad de los diferentes grupos estudiantiles por el control territorial de su entorno, impidiendo que los de otros planteles transitaran por determinadas calles, presentándose incluso trifulcas masivas entre dos o más planteles, con heridos de gravedad.

Las concentraciones educativas ayudaron a transformar el paisaje de los barrios. Usualmente el habitante del barrio se sentía dueño y confiado en su territorio. Con las migraciones, la llegada y tránsito de personas desconocidas por este territorio que siempre fue seguro, las expresiones, primero de inseguridad, posteriormente de violencia, van invadiendo los espacios.

La mayoría de los barrios populares fueron transformándose. Es común que en casi todos ellos se forme un sector comercial o una calle que concentra un mayor número de negocios. Otros

42 La metrópoli bogotana ha "conurbado" a varios municipios de origen precolombino que se transformaron en parte de la ciudad, como en el caso de Fontibón, Usme, Usaquén, Suba, Bosa, Engativá. Están muy cerca de ser anexados Soacha, Chía, Cota, entre otros. 43 Nombre que se le da a sitios clandestinos de baile para menores donde usualmente se consume alcohol y en algunas ocasiones drogas. 
incluso llegan a concentrar pequeñas empresas comerciales, industriales, talleres automotrices, de bicicletas, lo que atrae paulatinamente otro tipo de población, a menudo extraña a los habitantes originarios. Además, la mayoría de los barrios han sido impactados por obras de infraestructura urbana que alteran su tranquilidad.

El ambiente de pobreza y desempleo crónico, la informalidad, el ausentismo escolar, hacen que los barrios paulatinamente alberguen poblaciones que van invadiendo las calles de manera permanente, especialmente ventas ambulantes, y por jóvenes que poco a poco van entablando usos del espacio que pueden dar origen a conflictos con otros sectores de la comunidad barrial, hasta conformar situaciones extremas que conducen a la formación de escenarios violentos en la antiguamente idílica comunidad.

En algunas localidades encontramos un grave deterioro social y los oficios de muchos de los padres se ubicaban en la economía informal, en ocasiones asociado a actividades delincuenciales como el pequeño robo, el atraco, el microtráfico de estupefacientes. Por ejemplo, vendedores ambulantes o en los buses, como fachada, pero acompañados de las actividades mencionadas. No obstante, hay que señalar que la gran mayoría de los padres de familia se esfuerzan por una educación para que sus hijos sean "gente de bien".
Pero esos pequeños mercados de ilegalidad también penetran en la escuela, especialmente de dos formas: el pequeño robo de objetos de cada vez más valor comercial, con la generalización del uso de celulares y objetos electrónicos, principalmente, y los objetos de consumo tales como drogas y sustancias volátiles, especialmente pegantes de bajo precio que se aspiran y producen efectos psicoactivos.

El barrio alrededor de las escuelas se vuelve el escenario de mercados y circuitos legales e ilegales que se vinculan en torno a ellas, que por su tamaño y complejidad en la ciudad posmoderna, se convierte en el espacio articulador de los conflictos que se desarrollan en el barrio: las tribus, las pandillas, los combos, las barras de fútbol, entre otras, se concentran alrededor de la escuela. Esta se transforma en la articuladora de todos estos fenómenos urbanos y fácilmente se crea el clima para que dichos intercambios se tornen violentos. Hemos encontrado alrededor de la escuela los sitios y las prácticas que permiten que estos mercados y circuitos se realicen. En algunas tiendas de barrio muy cerca a las escuelas o en el centro de la ciudad, los jóvenes lo saben, se encuentran los sitios donde pueden vender a menos precio los objetos que salen de sus crecientes conductas predatorias al interior de la escuela, o afuera de ella, para obtener el dinero con el que tempranamente pueden satisfacer sus deseos de todo tipo de 
consumos: la ropa de marca, las maquinitas tragamonedas, las sustancias adictivas o el alcohol, que cada vez son más frecuentes entre ellos.

Los espacios comunitarios de padres, madres, maestros y líderes de las comunidades barriales pueden optar por un barrio capaz de contener a todos sin que los conflictos, normales y necesarios en toda comunidad humana, se transformen en actos violentos, previniendo los ambientes que propician los escenarios indeseables que dan lugar a espacios para la delincuencia juvenil, las confrontaciones violentas y los excesos que dañan la convivencia.

La familia, la escuela y el barrio, como comunidades dinámicas, tienen las capacidades para actuar preventivamente. Necesitan únicamente las herramientas adecuadas para que esta estrategia se construya. Y el punto de partida proponemos que sea la escuela, porque ella en sí misma cuenta con la estructura y la capacidad de convocatoria y de acción con que otras instancias e instituciones no cuentan. Ella ocupa un lugar central dentro de la comunidad.

\section{Conclusiones}

Las violencias sociales están inmersas en contextos sociales, económicos, políticos y planetarios. La metrópoli posmoderna es el teatro de todas las violencias. Unas están inmersas en dinámicas macrosociales cuyos alcances escapan a la capacidad de prevención comunitaria. Pero las violencias con dinámicas microsociales son posibles de intervenir y prevenir desde las mismas estructuras micro. Para ello es necesario que la escuela y la familia acepten que el mundo de los jóvenes cambió. Que los adultos, incluidos padres y maestros, no estamos preparados para los cambios que la infancia y la juventud han tenido en todos los aspectos en medio de una revolución cultural de carácter global. Que estamos viviendo un ciclo nuevo de las revoluciones de los jóvenes donde es cada vez más temprana la autonomización de los adolescentes. Y que si queremos transformar las situaciones de riesgo, tenemos el imperativo de comprender más y mejor esos cambios, respetar su autonomía como sujetos y colectivos, estigmatizar menos sus formas de vida y construir en común las soluciones. De lo contrario, ellos se alejarán del mundo de sus mayores y cada vez contarán con más recursos y argumentos para construir su mundo asumiendo el costo y los 
riesgos, como siempre ha sucedido en las luchas generacionales, y el resultado puede ser el menos deseable para la familia, para la escuela y para la sociedad.

Dentro de nuestro modelo teórico de comprensión de la violencias, planteamos dos grandes campos de violencias: macro y microsociales, donde, por acción de estos cambios acelerados y las situaciones endémicas de las ciudades latinoamericanas, se hace cada vez más compleja y borrosa la frontera entre los dos campos. La expansión de la pobreza, la informalidad y las economías ilegales, han creado climas propicios para que esas fronteras sean cada vez más difusas. En otras palabras, las diferentes formas de violencia se conectan cada vez más y se retroalimentan.

Nuestra hipótesis al respecto es la siguiente: la conexión más eficiente en la sociedad contemporánea entre las violencias macro y micro son las múltiples expresiones de violencia juvenil, dadas por sus estructuras móviles y semiorganizadas que fácilmente derivan en situaciones permanentes o esporádicas de delincuencia que las hace proclives a verse involucradas en circunstancias violentas. Lo que a su vez las coloca en la riesgosa frontera de las violencias organizadas.

Con altísima frecuencia la población latinoamericana tiene que sobrevivir del denominado "rebusque", nomadismo laboral informal y de desempleo disfrazado, que impide que las familias tengan un proyecto de vida estable y donde los jóvenes llevan la peor parte. Estas circunstancias han hecho que los niños y niñas de los sectores más pobres acompañen a los padres y hermanos en actividades callejeras donde se dan aprendizajes indeseables que facilitan el paso de la informalidad a la ilegalidad.

La ausencia de "inserción estable" en el mundo laboral crea las condiciones para la trashumancia, que hace vulnerables a todas las personas pero especialmente a los jóvenes. De otra parte, la debilidad de las instituciones se traduce en vacíos locales de gobierno en los barrios populares de las grandes ciudades, vacíos que se forman a raíz de una prolongada ausencia de las autoridades, lo cual permite que actores armados privados e informales suplan la presencia del Estado. Así bandas, pandillas o las maras se apropian de dichos territorios y encuentran en actividades ilegales, especialmente en el microtráfico, el mecanismo para su autonomía económica del mundo del trabajo. Estas circunstancias empoderan a muchos jóvenes que por esta vía serán importantes, ganándose un lugar que la ciudad les negó.

La exclusión, la discriminación, son la otra cara de los círculos viciosos de la pobreza y la violencia. Los jóvenes de los barrios más pobres conviven cotidianamente con los riesgos de violencia, drogadicción, promiscuidad, prostitución, atraco, 
y su inserción laboral exitosa es menos probable dado que la deserción escolar es frecuente y si un joven logra terminar la secundaria es difícil que alcance la educación superior, debido a las bajas tasas de cobertura en Colombia.

Los países latinoamericanos que han transitado por largas guerras civiles tienen fuertes secuelas o "herencias de la guerra", máxime en el caso colombiano que vive una de las más largas del planeta aún sin resolver. La reinserción sin prevención es más riesgosa que la misma guerra. Una paz mal hecha es tan mala como la guerra misma. Las posguerras son un camino de grandes dificultades donde las secuelas de la guerra no terminan cuando llega la paz. La elevación de la delincuencia urbana en Colombia, el rearme de la llamadas "bandas emergentes", la reconstitución de los ejércitos narcoparamilitares, aunque sin la misma fuerza, son una realidad con la que la ciudad colombiana tiene que convivir.

En virtud de la política prohibicionista y la guerra contra las drogas, la política criminal de los países productores equivocadamente ha puesto el foco de manera exagerada en la persecución a los delitos asociados, con un evidente desgaste. Los negocios transnacionales no disminuyen el flujo de cocaína hacia los Estados Unidos o Europa: por el contrario, el consumo ha aumentado sustancialmente. $Y$ el prohibicionismo causa efectos perversos en los precios ayudando a mantener exorbitantes tasa de ganancias.

La ciudad latinoamericana tiene unas características que la hacen distinta a las ciudades del primer mundo: 1 . receptoras de múltiples actores de los diferentes conflictos armados, 2. la exclusión social, la pobreza y la discriminación 3. la informalidadilegalidad, 4. la ineficacia y la corrupción. Estas características la convierten fácilmente en territorio de conflictos

En síntesis, en estas reflexiones hemos visto que estas situaciones violentas se inscriben en contextos mundiales asociados a fenómenos planetarios que se relacionan con los acelerados cambios presentados en el mundo del trabajo, la informalidad laboral que fácilmente prohíja la ilegalidad y la autonomización de la juventud, fenómenos que imprimen cambios sociales acelerados para los que las sociedades no estaban preparadas y con los cuales tendremos que aprender a convivir. 


\section{Referencias}

Álvarez, C. (2007). Hurto a residencias en Bogotá 1999-2006 -Estudio exploratorio. Bogotá: Secretaría de Gobierno de Bogotá D.C., Observatorio de Convivencia y Seguridad Ciudadana, SUIVD Sistema Unificado de Información de Violencia y Delincuencia. Ubicado en: www. ceacsc.gov.co/index. Consultado en octubre 1 de 2011.

Acnur. Desplazamiento interno en Colombia. En línea. Consultado el 19 de septiembre de 2011. Disponible en: http://www. acnur.org/t3/operaciones/situacion-colombia/lineas-estrategicas-y-objetivos-del-acnur/

Behrman, J., Duryea, S. y Székely, M. (2003). Aging and Economic Opportunities: What Can Latin America Learn from the Rest of the World? En: The Family in Flux: Household Decision-Making in Latin América. Washington: IDB.

Castells, M. (1995). La ciudad informacional. Madrid: Alianza Editorial.

Castells, M. (2006). La era de la información: economía, sociedad y cultura. México: Siglo XXI Editores. Quinta Edición. Vol. III.

Cepal (2008). Panorama social de América Latina. Apoyada por fuentes de la Organización Panamericana de la Salud, OPS, Estadísticas de la salud en las Américas. Ediciones 2003 y 2006.
Instituto Nacional de Medicina Legal y Ciencias Forenses (2001). Guía práctica para el dictamen de lesiones personales [En línea]. Bogotá: Panamericana. [Consultado 17 Febrero 2011]. Disponible en: <http://www. medicinalegal.gov.co $>$.

Instituto Nacional de Medicina Legal y Ciencias Forenses. Forensis. (2005). Datos para la vida. Bogotá, D. C.: Imprelibros S.A. Instituto Nacional de Medicina Legal y Ciencias Forenses. Forensis. (2006). Datos para la vida. Bogotá, D. C.: Montes S.A.

Instituto Nacional de Medicina Legal y Ciencias Forenses. Forensis. (2007). Datos para la vida. Bogotá, D. C.: International Print Ltda.

Instituto Nacional de Medicina Legal y Ciencias Forenses. Forensis. (2008). Datos para la vida. Bogotá, D. C.: Quebecor.

Debarbieux, E., Garnier, A., Montoya, Y. y Tichit, L. (1999). La violence en milieu scolaire: Vol. 2. Le désordre des choses. Paris: ESF éditeur.

Drucker, P. (1969). La era de la discontinuidad. Londres: William Heinemann Ltd.

Drucker, P. (1993). La sociedad poscapitalista. Barcelona: Apóstrofe. Hernández, M. 'En 2010, la cifra de colombianos residentes en el exterior llegará a los seis millones'. Comunicado de prensa. Colombianos en Nueva York. 5 de enero de 2010. En línea: 
http://www.conexioncolombia.com/Comunicado-enero5-2010.pdf. P. 1. Consultado el 20 de septiembre de 2011. Instituto Popular de Capacitación IPC, Corporación Jurídica Libertad (2010). Memoria de la impunidad en Antioquia: Lo que la justicia no quiso ver frente al paramilitarismo. Medellín: IPC. Corporación Jurídica Libertad. En línea. Disponible en: http://www.uclm.es/fundacion/jornadasCooperacion/pdf/enlaces/MemorialonpunidadAntioquia.pdf. Consultado el 10 de septiembre de 2011.

Jaramillo, J. (1996). El campo urbano-popular: poblamiento, espacio público, organizaciones dinamizadoras, tiempo libre. Bogotá: Universidad Nacional de Colombia.

Guerrero, J. (1996). Los antecedentes internacionales de la crisis colombiana. En: Pensamiento y acción. Colombia: UPTC, vol. 1: págs: 7-14.

Guerrero, J. (2002). Mercados de violencia y guerra civil en América Latina en los 90. En: Apuntes del Cenes. UPTC, vol. 22 págs: 263-293.

Kaplan, C. (2006). La inclusión como posibilidad. Buenos Aires: Ministerio de Educación, Ciencia y Tecnología. Organización de los Estados Americanos. Agencia Internacional para la Cooperación y el Desarrollo.

Ley 975 de 2005.

Lemus Murcia, O. (2007). Identificación, análisis y propuestas de políticas públicas para la prevención y control de las acciones de los ciudadanos que se desarrollan en torno al tránsito de la legalidad a la ilegalidad y viceversa en Bogotá. Bogotá: Secretaría de Gobierno de Bogotá D.C., Observatorio de Convivencia y Seguridad Ciudadana, SUIVD Sistema Unificado de Información de Violencia y Delincuencia. En línea. www.ceacsc.gov.co/index.

Llorente, M. V., y otros (2000). Violencia homicida y estructuras criminales en Bogotá. Bogotá: Universidad de los Andes.

Mesa, M. (2006). Violencia social y globalización en América Latina. Madrid: Fundación Carolina. Centro de investigaciones para la paz.

Monsiváis, C. (1999). Notas sobre violencia urbana. Revista Letras Libres [En línea], Mayo 1999, no. 5 [Consultado 25, septiembre, 2010] p. 34-39. Disponible en: < http://www. letraslibres.com/index.php?art=5795>. 
Mozzo, E. (2001). Capitalismo hoy: La nueva marginalidad. Acerca del libro Parias urbanos. Marginalidad en la ciudad a comienzos del milenio, de Loic Wacquant. En: Revista Herramienta. No. 16- http:// www.herramienta.com.ar/ revista-herramienta-n-16/capitalismo-hoy-la-nueva-marginalidad-acerca-del-libro-pariasde-la-ciudadm Consultado 05-01-2011.

Naciones Unidas. Directrices de las Naciones Unidas para la prevención de la delincuencia juvenil. (Directrices de Riad). Asamblea General. Resolución 45/112 del 14 de diciembre del 1990. En línea. http://www. ocavi.com/docs_files/file_696. pdf. Consultado 1 de octubre de 2011.

Organización Mundial de la Salud (2003). Informe mundial de violencia y de la salud.

Perea Restrepo, C. (2007). Con el diablo adentro: pandillas, tiempo paralelo y poder. México: Siglo XXI.

Reguillo Cruz, R. (2000). Emergencia de las culturas juveniles. Estrategias del desencanto. Bogotá: Grupo Editorial Norma.

Ruiz Botero, L. (2006). La escuela: territorio de frontera. Tipología de conflictos escolares según estudio comparado en Bogotá, Cali, Medellín. Medellín: Instituto Popular de Capacitación.
Rivera, S. y Barreto, L. La impunidad en el sistema penal acusatorio en Colombia. Ministerio del Interior y Justicia, Bogotá, Documento de trabajo, s.f. www. mij.gov.co/econtent/library/.../ DocNewsNo4362DocumentNo2463.PDFconsultado 07-012011

Roig-lbañez, J. (2006). La educación ante un nuevo orden mundial. Buenos Aires: Díaz de Santos Ediciones.

Romero, M. (Editor) (2007). La ruta de la expansión paramilitar y los acuerdos políticos. Bogotá: Corporación Nuevo Arco Iris, Cerec, Asdi.

Sassen, S. (2000). Cities in a world economy. Londres: Pine Forge Press.

Sennett, R. (2000). La corrosión del carácter. Las consecuencias personales del trabajo en el nuevo capitalismo. Barcelona: Anagrama. 